\title{
Evidence-Based Pediatric Outcome Predictors to Guide the Allocation of Critical Care Resources in a Mass Casualty Event
}

\author{
Philip Toltzis, $\mathrm{MD}^{1}$; Gerardo Soto-Campos, $\mathrm{PhD}^{2}$; Evelyn M. Kuhn, $\mathrm{PhD}^{3}$; Ryan Hahn, $\mathrm{DO}^{1}$; \\ Robert K. Kanter, $\mathrm{MD}^{4,5}$; Randall C. Wetzel, $\mathrm{MD}^{2,6}$
}

\begin{abstract}
Objective: ICU resources may be overwhelmed by a mass casualty event, triggering a conversion to Crisis Standards of Care in which critical care support is diverted away from patients least likely to benefit, with the goal of improving population survival. We aimed to devise a Crisis Standards of Care triage allocation scheme specifically for children.
\end{abstract}

Design: A triage scheme is proposed in which patients would be divided into those requiring mechanical ventilation at PICU

'Division of Critical Care, Department of Pediatrics, Rainbow Babies and Children's Hospital, Cleveland, $\mathrm{OH}$.

${ }^{2}$ Virtual PICU Systems LLC, Los Angeles, CA.

${ }^{3}$ National Outcomes Center, Children's Hospital of Wisconsin, Milwaukee, Wisconsin.

${ }^{4}$ Pediatric Critical Care Medicine, Department of Pediatrics, Virginia Tech Carilion School of Medicine, Roanoke, VA.

${ }^{5}$ National Center for Disaster Preparedness, Columbia University, New York, NY.

${ }^{6}$ Department of Anesthesiology Critical Care Medicine, Children's Hospital of Los Angeles, Los Angeles, CA.

This work was performed at the Rainbow Babies and Children's Hospital, Virtual PICU Systems, LLC, Children's Hospital of Wisconsin, and Children's Hospital of Los Angeles.

Supported, in part, through a contract with the Ohio Hospital Association. Presented, in part, at the Annual Congress of the Society of Critical Care Medicine, San Francisco, CA, January 2014.

Dr. Toltzis is employed by the University Hospitals of Cleveland/Case Medical Center. His institution received grant support from the Ohio Hospital Association/Ohio Department of Health. Dr. Kuhn has disclosed other support from VPS, LLC (contract between VPS, LLC and Children's Hospital and Health System). Dr. Kanter consulted for the National Center for Disaster Preparedness, lectured for the University of Michigan and the University of Oregon, and received support for travel from the National Center for Disaster Preparedness. His institution received grant support from the Baton Rouge Area Foundation. Dr. Wetzel received royalties from Elsevier, William and Wilkins, LWW (multiple textbook assignments); lectured for the University of Utah (Key Note Symposium Speaker and Visiting Professor); and received support for article research from the State of Ohio. His institution received grant support from the State of Ohio (research funding for developing triage schema) and the Whittier Foundation (research funding for VPICU). The remaining authors have disclosed that they do not have any potential conflicts of interest.

For information regarding this article, E-mail: philip.toltzis@uhhospitals.org

Copyright (C) 2015 by the Society of Critical Care Medicine and the World Federation of Pediatric Intensive and Critical Care Societies

DOI: 10.1097/PCC.0000000000000481 presentation and those not, and then each group would be evaluated for probability of death and for predicted duration of resource consumption, specifically, duration of PICU length of stay and mechanical ventilation. Children will be excluded from PICU admission if their mortality or resource utilization is predicted to exceed predetermined levels ("high risk"), or if they have a low likelihood of requiring ICU support ("low risk"). Children entered into the Virtual PICU Performance Systems database were employed to develop prediction equations to assign children to the exclusion categories using logistic and linear regression. Machine Learning provided an alternative strategy to develop a triage scheme independent from this process.

Setting: One hundred ten American PICUs

Subjects: One hundred fifty thousand records from the Virtual PICU database.

\section{Interventions: None.}

Measurements and Main Results: The prediction equations for probability of death had an area under the receiver operating characteristic curve more than 0.87 . The prediction equation for belonging to the low-risk category had lower discrimination. $R^{2}$ for the prediction equations for PICU length of stay and days of mechanical ventilation ranged from 0.10 to 0.18 . Machine learning recommended initially dividing children into those mechanically ventilated versus those not and had strong predictive power for mortality, thus independently verifying the triage sequence and broadly verifying the algorithm.

Conclusion: An evidence-based predictive tool for children is presented to guide resource allocation during Crisis Standards of Care, potentially improving population outcomes by selecting patients likely to benefit from short-duration ICU interventions. (Pediatr Crit Care Med 2015; XX:00-00)

Key Words: intensive care unit length of stay; intensive care unit mortality; mass casualty; palliative care; pandemic preparedness; triage

lthough no regional or national emergency has ever
overwhelmed American hospitals in the era of modern
critical care medicine, federal planners assume the likeli- 
catastrophes (1). The recent outbreaks of H1N1 influenza, enterovirus D68, and Ebola highlight the possibility that the medical infrastructure can be overwhelmed by a wide-spread disaster. In a severe pandemic, patients requiring critical care could greatly exceed the available resources for many weeks. Crisis Standards of Care (CSC) (2), including critical care triage allocation that diverts resources to subpopulations likely to survive with relatively short periods of ICU support, may improve population outcomes compared with first-come first-served (or random) selection (3-5).

Identification of patients whose ICU admission will most likely contribute to the greatest number of survivors at the population level depends on evidence-based, validated outcome predictors determined from patient characteristics at the time of presentation. A tool to predict survival of adult populations using a single arbitrary triage threshold has been incorporated in adult critical care triage guidelines proposed by professional organizations and public health planning agencies (5-14). No population outcome predictors have been validated as a triage tool for children. Furthermore, the proportion of critically ill children in typical PICUs who are at very high risk of mortality is so small that exclusion of such patients would scarcely alter resource availability and thus would achieve little advantage in population outcome. The identification of patients likely to survive with brief ICU support is necessary to gain greatest advantage in a triage allocation scheme for sustained emergencies, and there is no such validated tool for children or adults $(4,5,14)$

Recognizing these shortcomings, the Ohio Department of Health solicited aid in proposing a conceptual framework for a pediatric triage algorithm in the event of an overwhelming mass casualty and in deriving pediatric population outcome prediction equations to identify critically ill children likely to survive with brief critical care support. After devising a CSC allocation decision tree, these equations were derived from data from actual PICU admissions obtained from a large pediatric critical care registry. The details of this work are presented herein.

\section{MATERIALS AND METHODS}

\section{Primary Assumptions}

We made three assumptions in deriving our CSC resource allocation scheme:

1. Some children who present to the ICU during CSC will not require ICU care.

2. Some children will have a significant likelihood of dying despite ICU care.

3. Some children, although likely to benefit from critical care, would remain in the ICU consuming resources for a prolonged period of time, thus preventing care for others more likely to benefit with less resource utilization.

Thus, we developed prediction models to assign children presenting for critical care to three categories:

1. Excluded from PICU admission because they are likely to be too healthy to require ICU care.
2. Excluded from PICU admission and assigned to palliative care because they are likely too ill or too resource consuming.

3. Optimal for critical care because they are most likely to benefit with reasonable resource utilization.

\section{Triage Schemes}

We imagined a CSC triage scheme in which children are evaluated for PICU admission as they present to the hospital (Fig. 1). In this scheme, the first division is between children who are in respiratory failure (hereafter referred to as "intubated," whether actually intubated on PICU presentation or immediately requiring intubation) and those who are not, assuming that the presence of respiratory failure at admission will be the first factor influencing triage and because ventilators are likely to be a limited resource. The branching scheme thereafter divides casualties into the final three categories: "high risk-exclude/palliative care," "low risk-exclude," and "optimal-admit," conceptually similar to CSC triage schemes that have been proposed for adult patients (14). Intubated children are designated as "high risk" if they exceed a predetermined threshold for risk of mortality or if they are predicted to be resource consumptive by thresholds for PICU length of stay (LOS) or days of mechanical ventilation (DMV). Prolonged LOS was included along with DMV as an exclusion criterion in the event that ICU support modalities other than mechanical ventilation, such as hemodynamic support or response to rapidly evolving organ failure, prove unavailable in areas outside the ICU, a consideration included in some adult schemes $(5,14)$. Nonintubated children are subsequently evaluated regarding whether they are "low risk," defined a priori as a composite outcome of predicted mortality less than $0.5 \%$ combined with predicted 0 DMV and PICU LOS less than 3 days. Such children would be excluded as being too healthy for ICU admission. The remainder of nonintubated children is then divided in a similar fashion to that described above, depending on whether they exceed the predetermined high-risk thresholds for mortality, LOS, and DMV. Children not excluded by any of these criteria are designated as optimal for critical care (most likely to benefit without excessive resource utilization) and admitted to the PICU.

The triage scheme was designed so that it could be modified according to the nature of the mass casualty occurrence. In the event of an overwhelming pandemic causing a high prevalence of respiratory failure, for example, PICU admission likely would be confined exclusively to those requiring mechanical ventilation. The larger scheme thus would be simplified to employ only selected categories (high mortality, LOS, or DMV) among those presenting in respiratory failure (Fig. 2A). In a sudden-event disaster, characterized by a shortterm catastrophe affecting many victims limited to a discrete location and time, the triage scheme could be simplified further to consider only intubated patients and only on the basis of risk of mortality (Fig. 2B), assuming that resources outside the affected geographical area could be mobilized sufficiently quickly that prolonged LOS and DMV would not preclude PICU admission. 


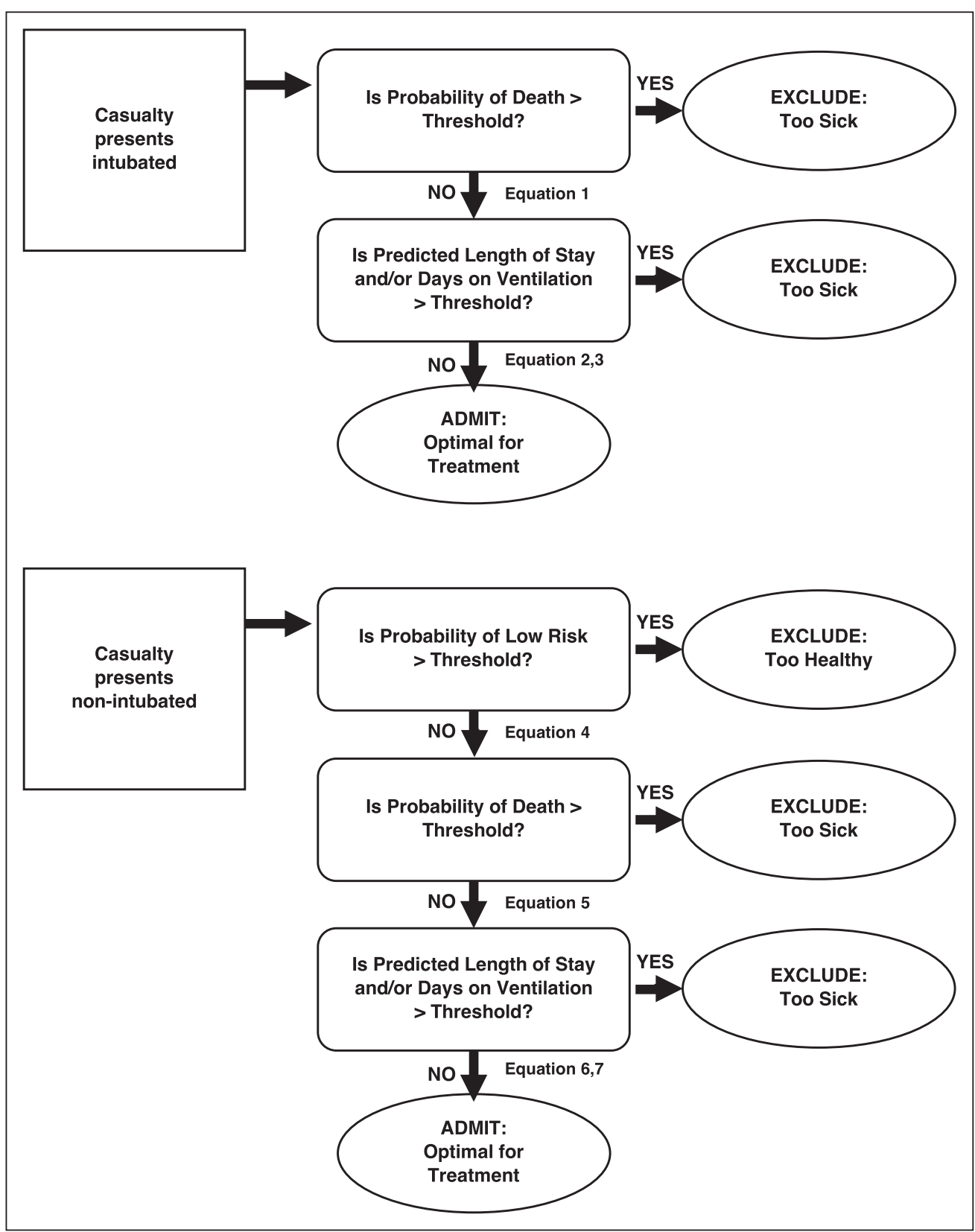

Figure 1. Illustration of the pediatric Crisis Standards of Care triage algorithm. The scheme initially divides children into those presenting to the PICU who are in respiratory failure and those who are not. Patients subsequently are excluded from PICU admission if they exceed a predetermined threshold for "high risk" (i.e., probability of death, PICU length of stay, or duration of mechanical ventilation) or "low risk" (i.e., mortality $<0.5 \%$, length of stay $<3 \mathrm{~d}$, and duration of mechanical ventilation, $0 \mathrm{~d}$ ). All the remaining children are deemed optimal for PICU treatment and admitted to the unit. The equation numbers next to each arrow refer to the prediction equations in Table 1.

\section{Development of Prediction Equations}

The datasets used to generate the prediction equations assigning children to each of the CSC exclusion categories were derived from the Virtual PICU Performance System (VPS). The VPS database is a prospective observational cohort of consecutive PICU admissions from a diverse set of hospitals caring for children in the United States. There is extensive quality validation performed by VPS staff prior to release of data for analysis. During the period of data extraction for the current project, there were approximately 110 participating PICUs with a database containing clinical data from over 600,000 pediatric admissions. Participating centers were required to enter extensive clinical information prompted by a uniform questionnaire on every PICU admission. Required elements included, among other variables, the established pediatric severity scores Pediatric Risk of Mortality (PRISM) II, PRISM III, and Pediatric Index of Mortality-2, entered by their component variables; primary and secondary diagnostic categories; and mechanical ventilator use, PICU LOS, and mortality.

To develop the prediction equations for each of the categories represented in the triage scheme, a randomly selected de-identified dataset of 150,000 VPS records was generated, representing patients who had been admitted between January 1, 2009, and December 31,2012 . We focused on variables available at PICU admission $(n=21$, as we envisioned a triage scheme that would be applied at the time of hospital presentation) that the investigators judged likely to be biologically associated with the outcomes. A 100,000-record subset (the development set) was used to derive the prediction equations. Univariate analyses were performed to test the association of candidate variables with the outcomes of interest (namely mortality, LOS, DMV, and low risk). Variables so identified were added in a stepwise manner to generate multivariate models. Logistic regressions were used to model the categorical bivariate outcomes mortality and low risk. Stepwise linear multivariate regressions were used for the logarithm of the continuous variables LOS and DMV. The $p$ value used to determine statistical significance in the stepwise regressions was 0.05 .

The remaining 50,000 records were used to validate the equations. The areas under receiver operator characteristic curves (AROC) were measured to determine the discriminative power of the logistic regressions. Decile and 20-quantile 

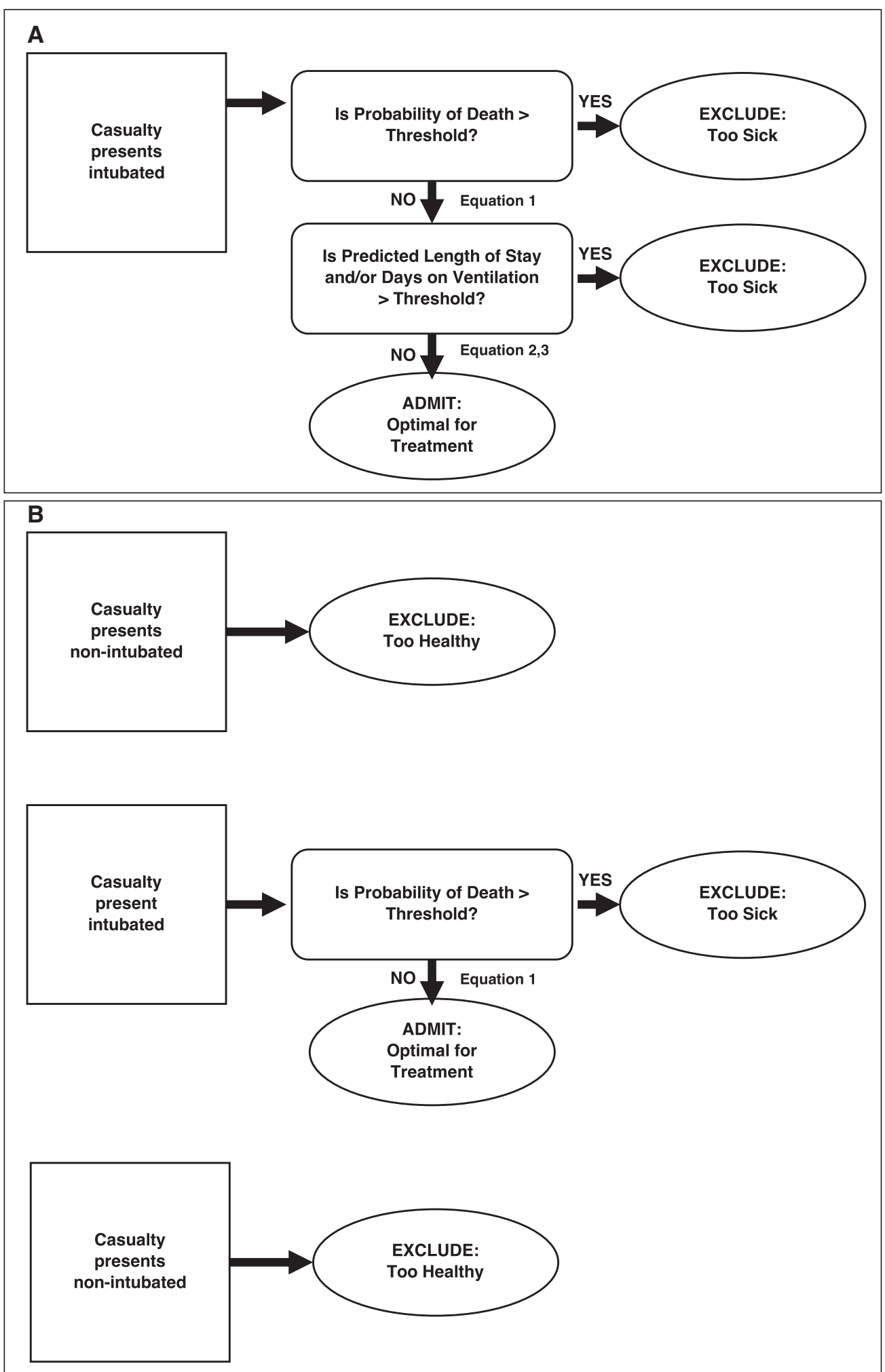

Figure 2. A, The algorithm presented in Figure 1 is modified to represent a scheme that can be used in a wide-spread pandemic of a respiratory virus. The scheme assumes that only children presenting to the PICU in respiratory failure will be candidates for PICU admission. Such children will be excluded from admission if they exceed a predetermined threshold for probability of death, PICU length of stay, or duration of mechanical ventilation. Children not requiring mechanical ventilation will be cared for elsewhere. B. The algorithm presented in Figure 1 is modified further to represent a scheme that can be used in a sudden-event catastrophe. As in Figure $2 A$, it is assumed that only children requiring mechanical ventilation will be considered for PICU admission. Children will not be excluded on the basis of prolonged length of stay or duration of mechanical ventilation, as it is assumed that resources can be quickly mobilized from neighboring regions not affected by the event. In both figures, the equation numbers next to each arrow refer to the prediction equations in Table 1.
Hosmer-Lemeshow tests (15) were used to evaluate the goodness-of-fit for the logistic regressions, and a $p$ value larger than 0.05 was considered a sign of a good fit. For the linear multivariate regressions, we computed unadjusted and adjusted correlations coefficients, $R^{2}$. All the analyses and models were done with version 2.15 .3 of the statistical software R ( $\mathrm{R}$ Core Team 2013, Vienna, Austria) (16) and independently verified with version 9.1 of SAS (Cary, NC).

\section{Machine Learning}

Because the development of a pediatric CSC triage scheme and the application of prediction equations to pediatric triage decisions are innovative and because they are difficult to confirm without actual disaster data, we sought another approach to compare to the logistic regression model based on the expert-determined decision tree described above. Machine learning (ML) is useful for the development of algorithms and has had extensive application for learning from large datasets both outside of and within healthcare (17-26). The technique has been applied to making triage decisions in the emergency department and renal transplantation and additionally in the development of triage tools for adult surge management (24-26). We used an ML approach similar to these (27-29) to provide an alternate strategy to designing a triage decision tree, which could be compared with the expertderived tree described above.

ML methodology was used to generate a decision tree learned using the same clinical and physiologic variables employed in the above prediction equations. A probabilistic decision tree (27) conditioned 


\section{Presents Intubated}

Equation 1: Mortality

$r=-3.860-0.780 \times$ ElectiveAdmit $-0.794 \times$

RecoveryFromSurgery $-1.484 \times$ NoHighRiskDx $+1.054 \times$ NoLowRiskDx $+2.840 \times$ PupilsNonReact $+0.022 \times$ SystBP_ forpim $+0.061 \times$ BaseExcess $+1.074 \times$ gcs_lt8 $+0.003 \times$ ageMonths $+0.533 \times$ under $1 \mathrm{yr}+0.820 \times$ categ_infec $0.220 \times$ categ_resp

$$
\begin{aligned}
& P_{\text {deathlnt }}=1 / 1+\exp (-r) \\
& \text { AROC }=0.869 ; \mathrm{H}-\mathrm{L}=0.182
\end{aligned}
$$

Equation 2: LOS

$\log$ LOSInt $=1.330-0.170 \times$ RecoveryFromSurgery -0.360 $\times$ NoHighRiskDx $+0.088 \times$ NoLowRiskDx $-0.650 \times$ PupilsNonReact $+0.003 \times$ SystBP_forpim $+0.023 \times \mathrm{FIO}_{2} /$ $\mathrm{PaO}_{2}+0.154 \times$ gcs_lt8 $+0.367 \times$ neonate $+0.346 \times$ under $1 \mathrm{y}$ $+0.370 \times$ categ_infec $+0.072 \times$ categ_card $-0.181 \times$ categ_inj $-0.347 \times$ categ_neur $+0.264 \times$ categ_resp

$\mathrm{LOS}_{\text {Int }}=\exp \left(\log \operatorname{LOS}_{\text {Int }}\right)$

Adjusted $R^{2}=0.101$

Equation 3: DMV

LogVentDaysInt $=0.845-0.072 \times$ ElectiveAdmit -0.554 $\times$ RecoveryFromSurgery - $0.252 \times$ AdmitAfterCardBypass $-0.530 \times$ NoHighRiskDx $+0.005 \times$ SystBP_forpim +0.030 $\times \mathrm{FlO}_{2} / \mathrm{PaO}_{2}+0.276 \times$ gcs_lt8 $+0.587 \times$ neonate $+0.460 \times$ under 1 y $-0.150 \times$ age 18 plus $+0.717 \times$ categ_infect -0.199 $\times$ categ_card $-0.452 \times$ categ_inj $-0.705 \times$ categ_neur + $0.492 \times$ categ_resp

VentDays $_{\text {Int }}=\exp ($ logVentDayslnt $)$

Adjusted $R^{2}=0.182$
Presents Nonintubated

Equation 4: Low risk

$r=1.465+0.330 \times$ ElectiveAdmit $+0.643 \times$

RecoveryFromSurgery - $0.391 \times$ AdmitAfterCardBypass

$+0.773 \times$ NoHighRiskDx $-0.958 \times$ NoLowRiskDx

- $0.007 \times$ SystBP_forpim $-0.956 \times$ gcs_lt8 -0.223

$\times$ neonate $-0.462 \times$ under 1 yr $-0.452 \times$ categ_infec

$-0.453 \times$ categ_card $+1.255 \times$ categ_inj $+0.246 \times$ categ_neur $-0.483 \times$ categ_

resp

$P_{\text {low-risk }}=1 / 1+\exp (-r)$

$\mathrm{AROC}=0.683 ; \mathrm{H}-\mathrm{L}<0.01$

Equation 5: Mortality

$r=-5.321-0.880 \times$ ElectiveAdmit $-1.860 \times$

RecoveryFromSurgery $-1.600 \times$ NoHighRiskDx +2.100

$\times$ NoLowRiskDx $+0.860 \times$ PupilsNonReact $+0.018 \times$

SystBP

forpim $+0.084 \times$ BaseExcess $+1.631 \times$ gcs_lt8 -

$2.314 \times$ categ_inf $-1.003 \times$ categ_neur

$P_{\text {deathNonlnt }}=1 / 1+\exp (-r)$

$\mathrm{AROC}=0.871 ; \mathrm{H}-\mathrm{L}=0.265$

Equation 6: LOS

$\log \mathrm{LOSNonInt}=0.453-0.160 \times$ ElectiveAdmit

- $0.180 \times$ RecoveryFromSurgery $+0.232 \times$

AdmitAfterCardBypass $-0.405 \times$ NoHighRiskDx +

$0.360 \times$ NoLowRiskDx $-0.004 \times$ SystBP_forpim +

$0.406 \times$ gcs_It8 $+0.0003 \times$ ageMonths $+0.210 \times$

neonate $+0.242 \times$ under $1 \mathrm{yr}+0.250 \times$ categ_infec

$+0.191 \times$ categ_card $-0.470 \times$ categ_inj $-0.070 \times$ categ_

neur $+0.207 \times$ categ_resp

$\mathrm{LOS}_{\text {Nonlnt }}=\exp (\log \mathrm{LOSNonlnt})$

Adjusted $R^{2}=0.101$

Equation 7: DMV

logVentDayNonlnt $=1.134-0.573 \times$

RecoveryFromSurgery $-0.795 \times$ AdmitAfterCardBypass

$-0.427 \times$ NoHighRiskDx $+0.447 \times$ under $1 y r+0.619 \times$

categ_infect $-0.560 \times$ categ_inj $-0.183 \times$ categ_neur +

$0.550 \times$ categ_resp

VentDays $_{\text {Nonlnt }}=\exp ($ logVentDaysNonlnt $)$

Adjusted $R^{2}=0.100$

ElectiveAdmit $=$ admission could have been postponed $>6 \mathrm{hr}$ without causing harm, RecoveryFromSurgery $=$ recovery from surgery principal reason for PICU admission, NoHighRiskDx = none of the following: cardiac arrest immediately preceding admission, severe combined immunodeficiency, leukemia or lymphoma after first induction, spontaneous cerebral hemorrhage, cardiomyopathy, HIV infection, liver failure as the principal reason for admission, or neurodegenerative disorder, NoLowRiskDx = none of the following: asthma, bronchiolitis, croup, obstructive sleep apnea, or diabetic ketoacidosis, PupilsNonReact = both pupils nonreactive during first hour of PICU admission, SystBP_forpim = first measured systolic blood pressure in first hour of PICU admission, subtracted from 120 , BaseExcess = base excess, gcs_It8 = Glasgow Coma Scale $<8$, ageMonths = age in months at admission, under1yr = age $<1$ yr at time of admission, categ infect, categ_resp, categ_card, categ_inj, categ_neur = diagnosis is infectious, respiratory, cardiac, injury, or neurologic, respectively, AROC = area under the receiver operating characteristic curve, $\mathrm{H}-\mathrm{L}=$ Hosmer-Lemeshow calculation for calibration, LOS $=$ length of stay, neonate $=$ age $<1$ mo at admission, $\mathrm{DMV}=$ days of mechanical ventilation, AdmitAfterCardBypass = admitted after operation using cardiac bypass, age 18 plus $=$ age $>18$ mo at admission.

on these variables was learned to predict the joint (combined) probability distributions of need for ventilation, mortality, LOS, and DMV. Briefly, we started with a single leaf (a tree with no decisions) that has a joint distribution over the outcomes and resources used for the entire population in the dataset. The next step was to consider splits of the data (decision nodes) that subdivide the population generating a tree with two leaves, testing all possible decisions. The model branch points (nodes) were based 
on the admission measurements. The decisions were scored and those yielding leaves with the best Bayesian score (the sum of the logs of the probabilities of the four outcome variables in each leaf adjusted for tree size) were used. The tree was learned in a standard greedy fashion (a recursive ML strategy that considers other solutions in the dataset), iteratively continuing to add splits until the Bayesian score of the resulting leaves was maximized and further splits did not increase the score (29).

We applied this unsupervised (no a priori assumptions of assignments or outcomes) decision tree, which captured potential nonlinear effects to provide probability estimates of the outcome distributions for given admission values for each patient. The final leaves of the decision tree represented the probabilities of the combined outcome groups: lived, not ventilated; lived, ventilate; died, not ventilated; and died ventilated. The leaf distribution so learned was a multinomial distribution (used to compute the probabilities in situations in which there are more than two possible outcomes) over the categorical outcomes of mortality and need for ventilation and a conditional log-normal distribution over the continuous outcomes of LOS and DMV. In this way, an optimal triage scheme was generated by ML from the data, without any presuppositions. Once the model was built on the training set, new patients (test set) were assigned to one of the leaves of the tree depending on their presentation values. In contrast to the triage model described above, the ML approach assigned the triage decisions based on a purely heuristic mathematical approach, free from expert opinion and modeled on the actual data to predict the probability of each outcome.

This project was approved by the VPS Research Committee and the Institutional Review Boards of Rainbow Babies and Children's Hospital/Case Medical Center, Cleveland, $\mathrm{OH}$, Children's Hospital of Wisconsin, and Children's Hospital of Los Angeles, who waived requirement for informed consent on the basis of nonhuman research.

\section{RESULTS}

\section{Prediction Equations}

The prediction equations for each of the triage exclusion categories are presented in Table 1. The AROC of the two mortality predicators (Eqs. 1 and 5 in Table 1 and Fig. 1, predicting mortality in patients arriving intubated and nonintubated, respectively) was more than 0.87 . Both equations also were well fitted according to the method of Hosmer and Lemeshow (Table 1). The equation predicting low risk (Eq. 4 in Table 1 and Fig. 1) had an AROC less than 0.70 and failed the test for goodness-offit. The adjusted $R^{2}$ for the equations predicting the continuous outcomes LOS (Eqs. 2 and 6) and DMV (Eqs. 3 and 7) in both the initially intubated and initially nonintubated patients ranged between 0.10 and 0.18 (Table 1 and Fig. 1).

\section{Selection of Exclusionary Thresholds}

Public health authorities recommend that plans for mass casualties include the establishment of Operations Command Units to continually monitor the availability or shortfall of medical resources before and after CSC has been declared (2). Once CSC has been initiated, adult triage schemes have recommended a single exclusionary mortality threshold $(90 \%)$ regardless of the degree of resource shortage (5). The currently proposed triage scheme recognizes that the balance between number of casualties and available resources during CSC will be different for different events and may even evolve during a particular mass casualty occurrence. Thus, the scheme allows the proportions of children excluded from PICU care ("high risk" plus "low risk") to be adjusted by manipulating the levels of risk of mortality and LOS or DMV (referred to as "thresholds") for assignment to the excluded categories, so the optimal treatment group can be sized to approximate the number of available beds. In Table 2 , example A, for example, the threshold probability of assignment to low risk was set to more than 0.60 (i.e., $>60 \%$ chance of being correctly assigned to the low-risk group) and of mortality to more than 0.70 (i.e., > 70\% chance of dying); for LOS or DMV, the threshold values were set to those that fell at or above the 90th percentile of the entire population. When these thresholds were applied to the total 150,000 record dataset, $69.9 \%$ of children were excluded from PICU admission. In Table 2, example $\mathrm{B}$, the threshold for assignment to the low-risk group was set more liberally than in example A, from more than 0.60 to more than 0.50 , with the other thresholds unchanged. This increased the proportion of children assigned to the low-risk group and consequently reduced the size of the optimal, admitted population from $30.1 \%$ to $26.0 \%$. In Table 2, example C, the threshold for assignment to low risk remained at more than 0.50 as in example B, but the threshold for risk of mortality and hence admission to the excluded high-risk group was reduced from more than 0.70 to more than 0.50 , and those for prolonged LOS or DMV were reduced from more than 90th to more than 80th percentile of the population. This adjustment resulted in expansion of the number of children assigned to the excluded high-risk cohort and therefore further reduction of the number assigned to the optimal admitted population. In this fashion, the proposed scheme permits adjustment of the thresholds to match variations in resource availability as the disaster evolves.

\section{Machine Learning}

The results from the ML experiments displayed two noteworthy properties, discovered independently from the above approach and without guidance (i.e., unsupervised ML from actual patient data). First, the most informative decision for triage assignment was whether the patient was intubated at the time of triage, thus agreeing with the expert-derived triage algorithm assumption. Second, the ML-predicted mortality among intubated patients generated an AROC of 0.81 , similar to that using logistic regression. The ML approach predicted DMV and LOS poorly for nonintubated children, again similar to the weaker predictive values of the regressions derived to predict category assignment of nonintubated children in the triage scheme. These ML results, taken together, independently tended to confirm the triage model derived from the first approach.

\section{DISCUSSION}

The Institute of Medicine (2) has recommended that the medical community preemptively prepares a response to future 


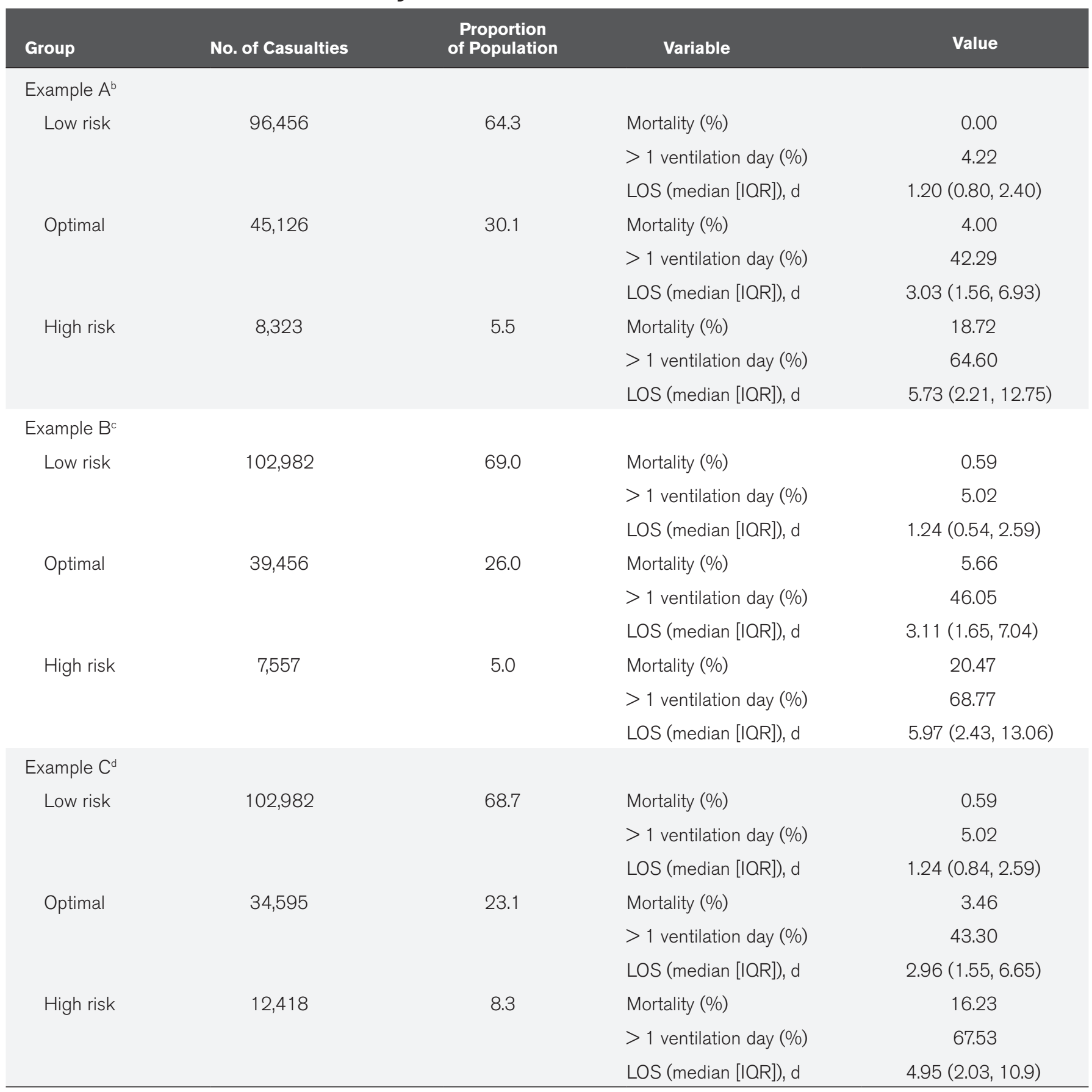

LOS $=$ length of stay, IQR = interquartile range.

aThe algorithm allows the proportions of children admitted to the PICU to be expanded or contracted based on the size of the mass casualty event and the relative shortfall of ICU resources, by adjusting the thresholds for assignment to each of the exclusion categories.

bThe proportions of children assigned to the high-risk, optimal, and low-risk categories if the thresholds are adjusted as follows: probability of low risk $>60 \%$, probability of death $>70 \%$, LOS and duration of ventilation $>90$ th percentile.

'The proportions of children assigned to the high-risk, optimal, and low-risk categories if the thresholds are adjusted as follows: probability of low risk $>50 \%$, probability of death $>70 \%$, LOS and duration of ventilation $>90$ th percentile.

dThe proportions of children assigned to the high-risk, optimal, and low-risk categories if the thresholds are adjusted as follows: probability of low risk $>50 \%$, probability of death $>50 \%$, LOS and duration of ventilation $>80$ th percentile.

mass casualties. These recommendations focus on developing plans to expand existing patient-care capabilities were a disaster to occur. In the most severe mass casualty events, however, it is anticipated that the need for critical care resources will be overwhelmed, prompting a conversion to CSC in which normally life-saving interventions will be diverted away from some patients who would have otherwise survived (2). Several schemes have been proposed to inform triage decisions in adult patients during CSC. The scheme proposed by a consensus group in Ontario (14), followed by a similar algorithm 
developed by experts convened by the United States Centers for Disease Control and Prevention (CDC) (5), recommends a two-step process in which, first, scarce resources are diverted from patients with predisaster chronic underlying conditions with limited potential for long-term survival. Patients who pass this step then are assessed for risk of mortality based on their initial and daily Sequential Organ Failure Assessment (SOFA) scores. Those whose SOFA scores predict a more than $90 \%$ mortality also are denied critical care (5).

These and similar adult-patient schemes are not readily adaptable to children. The exclusionary antecedent conditions are uncommon in children, and the ethics of such exclusions recently have been questioned even in adults (30). SOFA is not validated as a mortality prediction tool in the pediatric age group, and, in fact, existing data indicate that children with multiple organ dysfunction experience higher survival compared with adults with similar severity of disease (31); indeed, SOFA has been abandoned in recent revisions of even adult triage schemes (32). Furthermore, adult schemes do not assess for ICU resource overutilization. Based on these considerations, a task force of pediatric expert advisory to the CDC endorsed the concept of CSC ventilator and critical care triage allocation specifically for children (4). They declined to recommend any particular mass casualty triage criteria to guide PICU resource allocation, however, citing the need for empirical research to develop evidence-based quantitative tools to predict outcomes on the basis of early clinical characteristics (4).

We have proposed a mass casualty population-outcome predictive tool that addresses these issues, summarized as follows: 1) For the first time a pediatric-specific scheme has been developed, informed by prediction equations based on data from actual critically ill children entered into a current, large PICU database. 2) The decision-tree scheme was formulated by expert opinion and then largely confirmed in an entirely independent and unbiased fashion by ML. 3) Unlike adult schemes, the pediatric scheme considers the utilization of resources (LOS and DMV) as well as risk of mortality when considering PICU admission during CSC. 4) Unlike adult schemes, it does not exclude children based on predisaster comorbidities; antecedent conditions are relevant only if they affect mortality, LOS, DMV, and low risk. 5) The pediatric scheme allows the thresholds for PICU admission to be adjusted during CSC as the event progresses, to enable clinicians and public health officials to match changing casualty volumes to bed availability using criteria that could be employed simultaneously and consistently by all acute care facilities in the affected area. These steps were achieved all the while ensuring that the scheme satisfies key ethical principles pertaining to resource allocation during CSC $(7,30,33)$ : it is objective (using only measurable, clinically relevant data); is transparent; is egalitarian (assessing all children presenting for PICU admission equally); and guarantees procedural justice (by applying the same triage process consistently and to all comers).

The triage scheme assigns children to high-risk and lowrisk exclusion categories using physiologic variables available at PICU admission. Discrimination and goodness-of-fit of the mortality prediction equations, both for initially intubated and nonintubated children, were very strong. The predictive power of the other equations was not as compelling. Prediction of low risk was felt to be a necessary component of the CSC triage scheme, since conventional care practice frequently offers preemptive monitoring and interventions to children who are only moderately ill but whose illness may progress. Such children would not be appropriate for PICU admission during CSC. The AROC for assignment to the low-risk group, however, fell below 0.70 . Nevertheless, we reasoned that inaccurate assignment to this group was tolerable, since individual children misclassified as low risk and denied ICU support could be re-evaluated for PICU admission if their condition worsened, using the same criteria as other children vying for a PICU space at the same time. Additionally, the prediction equations with the continuous outcomes LOS and DMV were associated with low $R^{2}$ values. Others have demonstrated that models designed to predict ICU LOS in units serving adults are weak when they employ only variables collected early in the patients' course (34). Predictive power increases when data collected on subsequent ICU days are used (34), since these reflect the patients' response to initial interventions and early complications of critical care, events that are more reliably associated with subsequent need for ICU support. We made the deliberate decision to develop an algorithm that could be applied at the patient's PICU presentation because triage allocation decisions must be made at the time of admission.

The scheme is subject to several limitations. Common to all schemes that employ prediction scores derived from population analyses, the accuracy of the prediction for any individual patient is inexact. Although receiver operator characteristic curves summarize sensitivity and specificity of populationbased predictions across the entire range of predictive variable thresholds, sensitivity and specificity at particular thresholds cannot be derived reliably from receiver operator characteristic curves. Thus, although the AROC may appear satisfactory, sensitivity and specificity at particular thresholds are uncertain. Prediction scores perform better at the population level, however, and it is survival at the population level, rather than the individual, that is the focus of CSC. Second, the scores were derived from general PICU reference populations. It is possible that the performance of the prediction equations will be lower in a mass casualty event in which a large proportion of children will exhibit a particular clinical phenotype whose clinical severity may have different population distributions than reference populations. Established severity-of-illness scores, such as Acute Physiology and Chronic Health Evaluation and PIM, composed of physiologic and diagnostic categories similar to ours, perform well across a variety of illnesses, however. Furthermore, CSC prediction scores will need to assess outcome of children with conditions outside the one driving the mass casualty and thus should not be overfitted to any particular entity. Finally, all of the records in the VPS database are derived from children who received the full extent of available critical care. Altered capabilities during CSC may affect the accuracies of the prediction equations. 
The work proposed herein requires a demonstration that the algorithm results in greater survivorship in the affected population than a random assignment first-come first-served process. This test of efficacy, which can be gained only through a real disaster or sophisticated simulation, is critical, as a recent analysis suggests that an algorithm based on predictors with poor sensitivity or specificity may result in fewer survivors when compared with a random assignment strategy (35). Our group recently has performed preliminary experiments using computer simulation; these experiments have demonstrated that optimal thresholds for assignment to exclusion groups can be derived for a disaster of a given magnitude and that triage algorithms using these optimal thresholds outperform a first-come first-served strategy in terms of overall population survival (36). During an actual sustained emergency, however, it is mandatory that public health agencies responsible for implementing triage allocation assess the accuracy of outcome predictors as the situation evolves, potentially requiring revision of the prediction equations, based on real-time evidence from patients, patterns of care, and outcomes in the emergency.

\section{CONCLUSIONS}

An evidence-based predictive tool for children to guide resource allocation during CSC is presented, potentially improving population outcomes by selecting patient subpopulations most likely to benefit from ICU interventions. Next steps should include the development of a method to determine optimal triage thresholds on the basis of needs-to-resources ratio and validation of an algorithm that would incorporate population outcome predictors and optimal thresholds.

\section{ACKNOWLEDGMENT}

We gratefully acknowledge the contribution of Dr. Christian Shelton of the Computer Science Department of the University of California Riverside who provided extensive consultation concerning the machine learning approach undertaken in this study.

\section{REFERENCES}

1. Department of Health and Human Services: HHS Pandemic Influenza Plan. Washington, DC, US Department of Health and Human Services, 2005

2. IOM (Institute of Medicine): Crisis Standards of Care. A Systems Framework for Catastrophic Disaster Response. Washington, DC, The National Academies Press, 2012

3. Utley M, Pagel C, Peters MJ, et al: Does triage to critical care during a pandemic necessarily result in more survivors? Crit Care Med 2011; 39:179-183

4. Christian MD, Toltzis P, Kanter RK, et al; Task Force for Pediatric Emergency Mass Critical Care: Treatment and triage recommendations for pediatric emergency mass critical care. Pediatr Crit Care Med 2011; 12:S109-S119

5. Devereaux AV, Dichter JR, Christian MD, et al; Task Force for Mass Critical Care: Definitive care for the critically ill during a disaster: A framework for allocation of scarce resources in mass critical care: From a Task Force for Mass Critical Care summit meeting, January 26-27, 2007, Chicago, IL. Chest 2008; 133:51S-66S

6. Ferreira FL, Bota DP, Bross A, et al: Serial evaluation of the SOFA score to predict outcome in critically ill patients. JAMA 2001; 286:1754-1758

7. Powell T, Christ KC, Birkhead GS: Allocation of ventilators in a public health disaster. Disaster Med Public Health Prep 2008; 2:20-26
8. Monroe JA: Indiana State Department of Health pandemic influenza operations plan. Available at: http://www.in.gov/isdh/files/ PandemiclnfluenzaPlan.pdf. Accessed June 5, 2014

9. Ball R, Schneider P: South Carolina prepares for pandemic influenza: An ethical perspective. Available at: http://www.scdhec.gov/Health/ docs/ophp/SC $\% 20$ Prepares $\% 20$ for $\% 20$ Pandemic\%20Ethics $\% 20$ Paper\%2011_09_06\%20CF\%20(2).pdf. Accessed June 17, 2015

10. Pezzino G, Simpson SQ: Guidelines for the use of modified health care protocols in acute care hospitals during public health emergencies, Kansas. Available at: http://www.kdheks.gov/cphp/download/ Crisis_Protocols.pdf. Accessed June 5, 2014

11. Florida Department of Health: Pandemic influenza: Triage and scare resource allocation guidelines. Available at: http://home. smh.com/sections/services-procedures/medlib/Disaster_Ethics/ NLM/FDOH\%20Pandemic\%2OInfluenza\%20Triage\%20and\%20 Scarce\%20Resource\%20Allocation\%20Guidelines\%20April\%20 2010.pdf. Accessed June 5, 2014

12. Tennessee Department of Health, Pandemic influenza response plan. Available at: http://health.state.tn.us/ceds/PDFs/2006_PanFlu_Plan. pdf. Accessed June 5, 2014

13. Utah Hospitals and Health Systems Association: Utah pandemic influenza hospital and ICU triage guidelines. Available at: http://pandemicflu.utah.gov/plan/med_triage081109.pdf. Accessed June 5, 2014

14. Christian MD, Hawryluck L, Wax RS, et al: Development of a triage protocol for critical care during an influenza pandemic. CMAJ 2006; 175:1377-1381

15. Hosmer DW, Lemeshow S: A goodness-of-fit test for the multiple logistic regression model. Commun Stat 1980; A10:1043-1069

16. R Core Team: R: A Language Environment for Statistical Computing. Vienna, Austria, R Foundation for Statistical Computing, 2013

17. Paliouras G, Karkaletsis VS, Constantine D (Eds): Machine Learning and Its Applications. Heidelberg, NY, Springer Verlag Berlin, 2001

18. Cleophas TJ, Zwinderman AH: Machine Learning in Medicine. Berlin, Springer Verlag, 2013

19. Song X, Mitnitski A, Cox J, et al: Comparison of machine learning techniques with classical statistical models in predicting health outcomes. Stud Health Technol Inform 2004; 107:736-740

20. Jensen PB, Jensen LJ, Brunak S: Mining electronic health records: Towards better research applications and clinical care. Nat Rev Genet 2012; 13:395-405

21. Pearce CB, Gunn SR, Ahmed A, et al: Machine learning can improve prediction of severity in acute pancreatitis using admission values of APACHE II score and C-reactive protein. Pancreatology 2006; 6:123-131

22. Wu J, Roy J, Stewart WF: Prediction modeling using EHR data: Challenges, strategies, and a comparison of machine learning approaches. Med Care 2010; 48:S106-S113

23. Magoulas GD, Andriana P: Machine learning in medical applications. Lect Notes Comput Sc 2001; 2049:300-307

24. Dexheimer JW, Leegon J, Aronsky D: Predicting hospital admission at triage in an emergency department. AMIA Annu Symp Proc 2007; 937

25. Reinaldo F, Fernandes $C$, Rahman A, et al: Assessing the eligibility of kidney transplant donors. Lect Notes Comput Sc 2009; 5632:802-809

26. Triage tool for accurate disposition of patients in disaster response. Available at: http://www.pacercenter.org/research/triage-tool-foraccurate-disposition-of-patients-in-disaster-response.aspx. Accessed March 12, 2015

27. Buntine W: Learning classification trees. Stat Comput 1992; 2:63-73

28. Harrington P: Machine Learning in Action. Shelter Island, NY, Manning Publications, 2012

29. Fraley C, Raftery AE: Bayesian regularization for normal estimation and model based clustering. J Classif 2007; 24:155-181

30. White DB, Katz MH, Luce JM, et al: Who should receive life support during a public health emergency? Using ethical principles to improve allocation decisions. Ann Intern Med 2009; 150:132-138

31. Typpo KV, Petersen NJ, Hallman DM, et al: Day 1 multiple organ dysfunction syndrome is associated with poor functional outcome and mortality in the pediatric intensive care unit. Pediatr Crit Care Med 2009; 10:562-570 
32. Christian MD, Sprung CL, King MA, et al; Task Force for Mass Critical Care; Task Force for Mass Critical Care: Triage: Care of the critically ill and injured during pandemics and disasters: CHEST consensus statement. Chest 2014; 146:e61S-e74S

33. Biddison LD, Berkowitz KA, Courtney B, et al; Task Force for Mass Critical Care; Task Force for Mass Critical Care: Ethical considerations: Care of the critically ill and injured during pandemics and disasters: CHEST consensus statement. Chest 2014; 146:e145S-e155S
34. Kramer AA, Zimmerman JE: A predictive model for the early identification of patients at risk for a prolonged intensive care unit length of stay. BMC Med Inform Decis Mak 2010; 10:27

35. Kanter RK: Would triage predictors perform better than first-come, firstserved in pandemic ventilator allocation? Chest 2015; 147:102-108

36. Toltzis P, Gall C, Kolker A, et al: Optimal thresholds for a PICU ventilator allocation algorithm in a pandemic. Abstract 147. Congress of the Society for Critical Care Medicine, Phoenix, AZ, January 2015 